\title{
A Rare Fatal Bile Peritonitis after Malposition of Endoscopic Ultrasound-Guided 5-Fr Naso-Gallbladder Drainage
}

\author{
Tae Hyung Kim, Hyun Jin Bae and Seung Goun Hong \\ Department of Internal Medicine, SAM Anyang Hospital, Anyang, Korea
}

Endoscopic ultrasound (EUS)-guided gallbladder (GB) drainage has recently emerged as a more feasible treatment than percutaneous transhepatic GB drainage for acute cholecystitis. In EUS-guided cholecystostomies in patients with distended GBs without pericholecystic inflammation or prominent wall thickening, a needle puncture with tract dilatation is often difficult. Guidewires may slip during the insertion of thin and flexible drainage catheters, which can also cause the body portion of the catheter to be unexpectedly situated and prolonged between the GB and intestines because the non-inflamed distended GB is fluctuant. Upon fluoroscopic examination during the procedure, the position of the abnormally coiled catheter may appear to be correct in patients with a distended stomach. We experienced such an adverse event with fatal bile peritonitis in a patient with GB distension suggestive of malignant bile duct stricture. Therefore, the endoscopist should confirm the indications for cholecystostomy and determine whether a distended GB is a secondary change or acute cholecystitis. Clin Endosc 2020;53:97-100

Key Words: Bile peritonitis; Distension; Endoscopic ultrasound-guided gallbladder drainage; Indication

\section{INTRODUCTION}

Acute cholecystitis has traditionally been treated by laparoscopic cholecystectomy with very low complication rates. Percutaneous transhepatic gallbladder (GB) drainage is a relatively effective option and is widely used for acute cholecystitis before surgical treatment. Presently, it is often used for patients in palliative care with acute cholecystitis and malignant biliary strictures such as cholangiocarcinomas. Recent advances in endoscopic ultrasound (EUS)-guided procedures allows for successful and effective GB drainage. ${ }^{1}$ The complication rates for EUS-guided GB drainage and percutaneous GB drainage are similar; ${ }^{2,3}$ however, EUS-guided GB drainage

Received: January 16, 2019 Revised: May 23, 2019

Accepted: May 24, 2019

Correspondence: Seung Goun Hong

Department of Internal Medicine, SAM Anyang Hospital, 9 Samdeok-ro, Manan-gu, Anyang 14030, Korea

Tel: +82-31-467-9114, Fax: +82-31-449-0151, E-mail: permi@naver.com ORCID: https://orcid.org/0000-0001-6841-235X

(cc) This is an Open Access article distributed under the terms of the Creative Commons Attribution Non-Commercial License (http://creativecommons.org/ licenses/by-nc/3.0) which permits unrestricted non-commercial use, distribution, and reproduction in any medium, provided the original work is properly cited. can sometimes be challenging. Multiple procedures must be performed during the steep learning curve to master the technique. Although rare, serious complications, such as proximal migration of the inserted stent into the peritoneum, can occur. Such adverse events can be promptly treated with surgery or percutaneous drainage, depending on the patient's status. Naso-biliary drainage catheters are widely used for biliary decompression in endoscopic retrograde cholangiopancreatography, and the catheter is sometimes used for EUS-guided GB drainage. A 5-Fr catheter is more flexible and more comfortable for patients than a 7-Fr catheter; however, it is vulnerable to kinking and tangling during the procedure. Proper and potential indication for EUS-guided cholecystostomy in acute cholecystitis is very important because the procedure is often difficult in patients with non-inflamed, fluctuant, and distended GB. We experienced a case of prolonged drooping and coiling of the catheter between the GB and duodenum during the procedure. The position of the catheter was believed to be properly situated in the distended GB as shown by two-dimensional fluoroscopic images at that time. 


\section{CASE REPORT}

An 80-year-old female patient was admitted to our hospital presenting with yellow skin color and anorexia with abdominal pain for two weeks. Physical examination revealed right upper quadrant tenderness and equivocal Murphy's sign without other remarkable findings. Her initial vital signs were stable. The laboratory test results were as follows: white blood cell count, 4,900/ $\mathrm{mm}^{3}$; serum hemoglobin, $10.7 \mathrm{~g} / \mathrm{dL}$; aspartate transaminase, $205 \mathrm{U} / \mathrm{L}$; alanine transaminase, $254 \mathrm{U} / \mathrm{L}$; alkaline phosphatase, 1,259 U/L; total bilirubin, $8.9 \mathrm{mg} / \mathrm{dL}$; blood urea nitrogen/creatinine, $8 / 0.7 \mathrm{mg} / \mathrm{dL}$; amylase, $56 \mathrm{U} / \mathrm{L}$; and CA19-9, 22 U/mL. An abdominal computed tomography (CT) scan showed segmental enhanced distal bile duct stenosis with upstream bile duct dilation and GB distension suggestive of distal common bile duct malignancy (Fig. 1). The endoscopic retrograde cholangiogram showed similar bile duct findings. A biopsy with brush cytology (Boston Scientific, Natick, MA, USA) was performed for the distal bile duct lesion. Two 7-Fr pigtail plastic stents (Zimmon biliary stent; Cook Endoscopy, Bloomington, IN, USA) were inserted for biliary decompression. An EUS showed a distended GB with wall thickening consistent with acute cholecystitis. EUS-guided GB drainage was performed after puncture of the GB with a 19-gauge nee- dle (EchoTip-19; Cook Endoscopy, Winston-Salem, NC, USA) and tract dilatation by a needle knife with a 6-mm Hurricane dilation catheter (Hurricane RX balloon; Boston Scientific). A 5-Fr naso-biliary drainage catheter (ENBD-5; Cook Endoscopy, Winston-Salem, NC, USA) was then inserted because it could be easily removed without endoscopy (Fig. 2A-C). The body portion of the catheter was unintentionally prolonged with coiling between the GB and duodenum. At that time, we mistakenly concluded that the coiling of the catheter was well placed in the stomach lumen as shown by two-dimensional fluoroscopic imaging (Fig. 2D). The patient complained of severe abdominal pain after the procedure. An abdominal $\mathrm{X}$-ray showed diffuse free air and unintended prolonged malposition of the catheter between the GB and duodenum (Fig. $3 \mathrm{~A})$. The patient's vital signs were relatively unremarkable except for an intermittent fever with a temperature greater than $37.5^{\circ} \mathrm{C}$. The catheter continued to function and drain the bile. We explained the possible development of bile peritonitis due to malposition of the bile drainage catheter to the patient and her family and then recommended surgery. However, the patient and her family were reluctant to agree to surgery, and rather wanted conservative or other interventional treatments. The patient continued to experience abdominal pain with distension. A follow-up abdominal CT scan three days
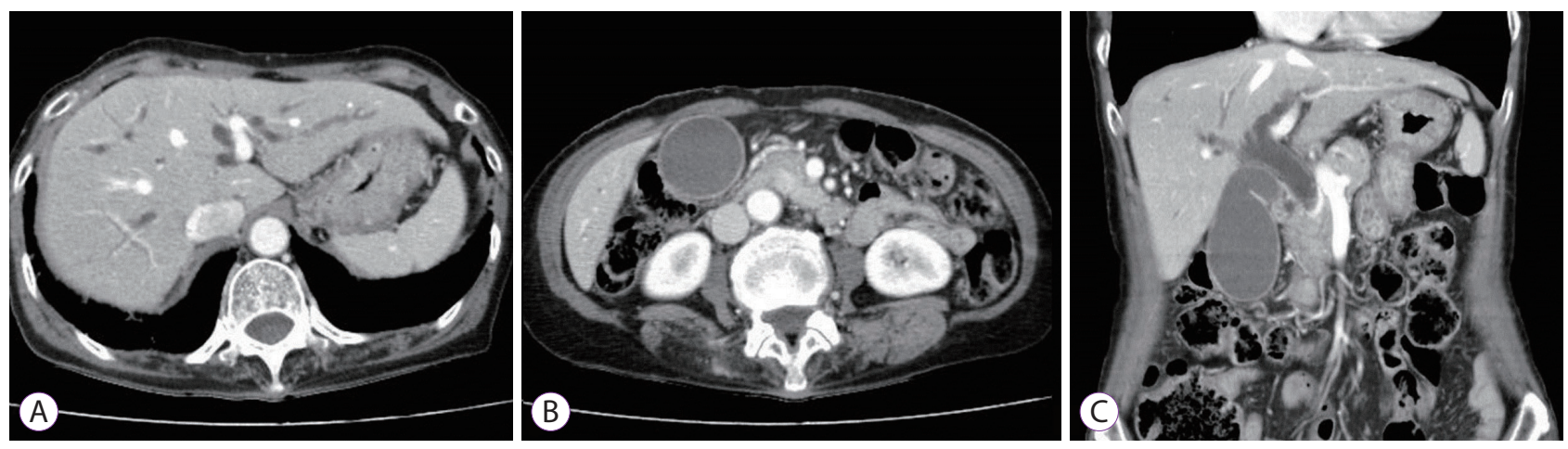

Fig. 1. Abdominal computed tomography scan showing that both intrahepatic bile ducts were dilated (A), and distension of the gallbladder with minimal wall thickening was observed (B). The coronal image shows an abrupt narrowing of the distal common bile duct suggestive of bile duct malignancy (C).
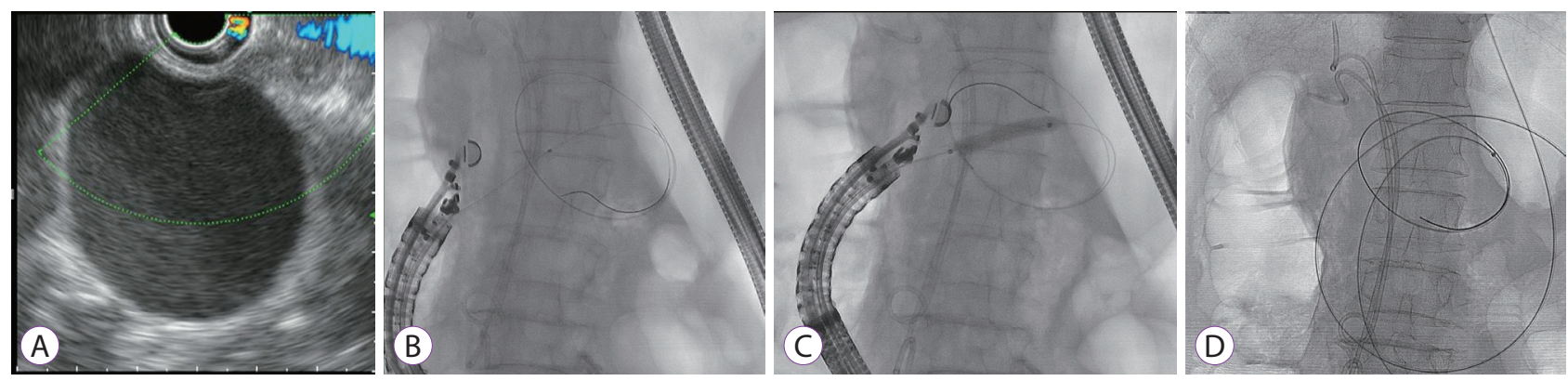

Fig. 2. Endoscopic ultrasound-guided gallbladder drainage. The endoscopic ultrasound shows a distended gallbladder with wall thickening (A). Tract dilatation was performed with a needle knife and 6-mm Hurricane dilation catheter consecutively $(B, C)$. Catheter coiling was presumed to be well situated in the stomach as shown by fluoroscopic imaging (D). 
later confirmed malposition of the catheter (Fig. 3B, C). The catheter was then removed even though it was functioning appropriately. The histological results were not consistent with bile duct malignancy and only showed inflammation. We inserted multiple drainage catheters to drain the bile-containing fluid collected in the abdominal cavity with percutaneous transhepatic GB drainage, accompanied by the administration of broad-spectrum antibiotics. However, the patient passed away due to sepsis accompanied by adult respiratory distress syndrome 35 days after admission.

\section{DISCUSSION}

EUS-guided cholecystostomy is recommended for patients with acute cholecystitis if they are unsuitable for emergent cholecystectomies, have received palliative treatment for advanced malignancies, or if percutaneous cholecystostomy is contraindicated. ${ }^{4}$ In our patient, despite the presence of a distended GB with wall thickening on the EUS image, the diagnosis of acute cholecystitis was indefinite because Murphy's sign was equivocal on the physical examination, she had vague non-specific pain or discomfort in her right upper quadrant area, GB distension without prominent wall thickening and pericholecystic inflammation were observed on the initial abdominal CT scan, and her initial white blood cell count was normal. In addition, the EUS and CT findings were presumably attributable to Courvoisier's sign, an enlarged and palpable GB in patients with obstructive jaundice mainly caused by a pancreato-biliary tumor. Thus, the diagnosis of acute cholecystitis in our patient could be incorrect and the cholecystostomy might have been unnecessarily performed in this patient. The main problem was bile leakage, which was related to the EUS-guided cholecystostomy due to a gap between the GB and fistulous tract. However, there was no significant bile leakage from the puncture site due to adherence of the inflamed GB wall to the adjacent gut wall that prevented leakage. ${ }^{5}$ Bile leakage and slippage of the guidewire during the procedure can occur, as seen in our patient, with GB distension without prominent pericholecystic inflammation or wall thickening.

A naso-biliary drainage catheter was used in our patient because of temporary insertion for distended GB. Many drainage devices have been developed for EUS drainage procedures. Besides the double-ended pigtail design of plastic stents, other stent modifications have recently been implemented to reduce biliary leakage and migration. Changes to both ends of the catheter using anchoring and apposing methods can decrease adverse events such as biliary leakage and stent migration. Among various modified stents, two specially designed covered metal stents include the anchoring-type at both ends and a lumen-apposing-type with a saddle-shaped design. The Bona-AL stent (Standard Sci Tech Inc., Seoul, Korea) $)^{6,7}$ and SPAXUS stent (Taewoong Medical, Gimpo, Korea) ${ }^{8,9}$ are often used in patients with acute cholecystitis during EUS-guided cholecystostomy. The Bona-AL stent is a fully covered metal stent that is $10 \mathrm{~mm}$ in diameter. It has anti-migration flaps on both ends. The SPAXUS stent is another fully covered metal stent with larger diameters (16 and $20 \mathrm{~mm}$ ). Both ends become lumen-apposing after deployment. The naso-biliary drainage catheters ${ }^{10-12}$ or 7 -Fr double-pigtail plastic stents ${ }^{13}$ have also been used in patients with acute cholecystitis before the development of metal stents, and the two plastic devices are used one-fourth as often among the patients in our hospital now. Two advantages of metal stents are larger diameters that allow good biliary drainage and less blockage and con-
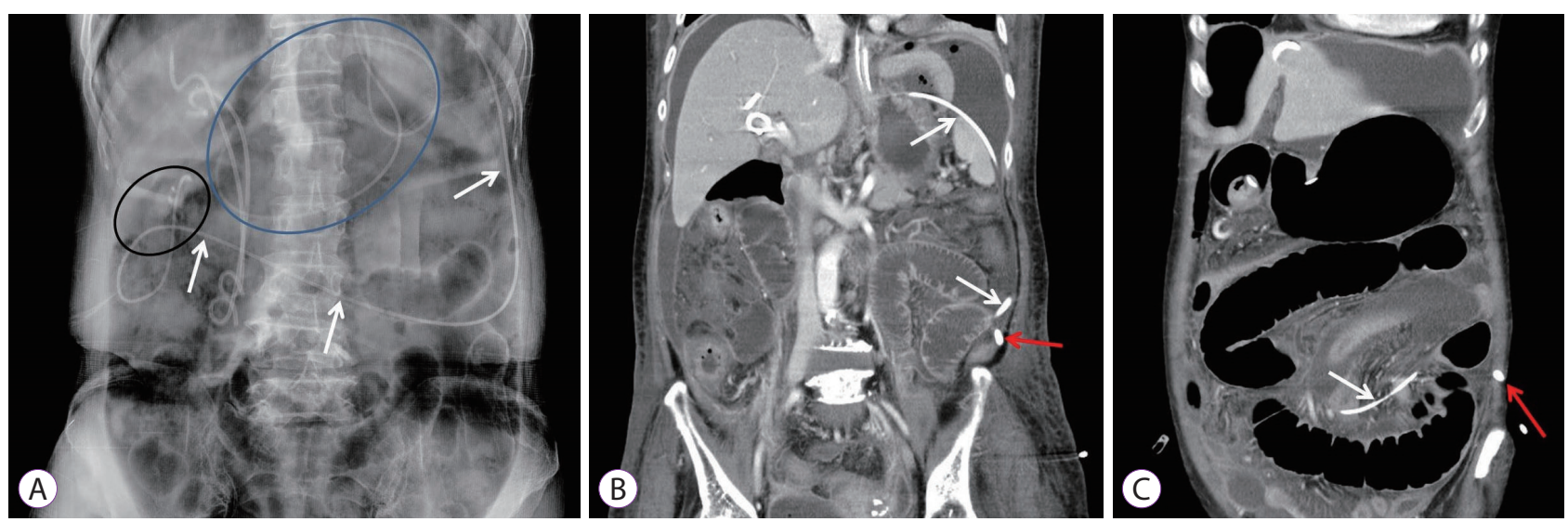

Fig. 3. Abdominal X-ray and follow-up computed tomography scan. The abdominal X-ray shows diffuse free air and marked prolonged malposition of the body of the 5-Fr naso-biliary catheter (white arrows) between the duodenum (blue circle indicating stomach) and gallbladder (black circle) (A). An abdominal computed tomography scan confirmed the prolonged malposition of the catheter in the peritoneal cavity (white arrows). A small portion of another drainage catheter in the left lower abdomen (red arrow) was observed, implanted to drain the fluids collected in the peritoneal cavity $(B, C)$. 
venient recapture of partially deployed stents that have been malpositioned during the procedure. Repositioning of the naso-biliary drainage catheter or plastic stent is very difficult or even impossible in cases where they are improperly positioned during deployment. Thus, the procedure is repeated, another puncture is made, and another guidewire is inserted into the GB.

Transpapillary insertion of the naso-biliary drainage catheter is often performed in patients with acute cholecystitis. ${ }^{14-16}$ However, in difficult cases, cystic duct cannulation can sometimes fail, and EUS-guided procedures can preclude these difficulties. A 5-Fr naso-biliary drainage catheter is very flexible and is softer than a 7-Fr catheter; therefore, it is more comfortable for the patient. However, during the insertion of a 5-Fr catheter into the GB, the force delivered over the guidewire through the dilated tract between the target and gastrointestinal tract is less than that in a case that uses a 7-Fr catheter in EUS-guided drainage procedures. Kinking and coiling of the catheter between the target and gastrointestinal tract caused by excessive pushing of the catheter can develop, making it difficult to recognize malposition of the catheter because coiling in the abdominal cavity can be misjudged as proper placement in a distended stomach via two-dimensional fluoroscopic imaging, as in the case described here. Previous studies have reported that pneumoperitoneum with biliary leakage rarely occurs from the use of naso-biliary drainage catheters. ${ }^{10,12}$

There were two additional clinical points during the procedure besides the incorrect indications for the procedure in this patient. The above adverse event could be attributed to the large dilatation made by the 6-mm biliary dilator balloon with a needle knife because the initial insertion of the 5-Fr catheter into the GB failed after tract dilatation by the needle knife. The failure of the first catheter insertion tract dilatation with only the needle knife was probably due to the fluctuation of the GB without prominent inflammation. The second point was that the attempt at an excessive deep insertion of the 5-Fr catheter into the GB for fear that proximal migration of the catheter can lead to malposition and catheter coiling between the GB and duodenum.

This case highlights the following: (1) the proper indication for EUS-guided GB drainage in patients with acute cholecystitis is very important and must be based on collective clinical and physical findings, (2) excessive dilatation of the tract between the intestine and GB should be avoided in cases where insertion of a 5-Fr naso-biliary drainage catheter is difficult, and (3) excessive pushing on the 5-Fr naso-biliary drainage catheter for deep insertion into the GB can lead to coiling and kinking outside the duodenum or stomach. Thus, endosco- pists need to cautiously confirm the indications for EUS-guided cholecystostomy in patients with GB distension and determine whether the status of the GB is only a secondary change or truly acute cholecystitis.

\section{Conflicts of Interest}

The authors have no financial conflicts of interest.

\section{REFERENCES}

1. Saumoy M, Novikov A, Kahaleh M. Long-term outcomes after EUS-guided gallbladder drainage. Endosc Ultrasound 2018;7:97-101.

2. Ahmed O, Rogers AC, Bolger JC, et al. Meta-analysis of outcomes of endoscopic ultrasound-guided gallbladder drainage versus percutaneous cholecystostomy for the management of acute cholecystitis. Surg Endosc 2018;32:1627-1635.

3. Tyberg A, Saumoy M, Sequeiros EV, et al. EUS-guided versus percutaneous gallbladder drainage: isn't it time to convert? J Clin Gastroenterol 2018;52:79-84.

4. Choi JH, Lee SS. Endoscopic ultrasonography-guided gallbladder drainage for acute cholecystitis: from evidence to practice. Dig Endosc 2015;27:1-7.

5. Ahmed O, Ogura T, Eldahrouty A, et al. Endoscopic ultrasound-guided gallbladder drainage: results of long-term follow-up. Saudi J Gastroenterol 2018;24:183-188.

6. Choi JH, Lee SS, Choi JH, et al. Long-term outcomes after endoscopic ultrasonography-guided gallbladder drainage for acute cholecystitis. Endoscopy 2014;46:656-661.

7. Jang JW, Lee SS, Park DH, Seo DW, Lee SK, Kim MH. Feasibility and safety of EUS-guided transgastric/transduodenal gallbladder drainage with single-step placement of a modified covered self-expandable metal stent in patients unsuitable for cholecystectomy. Gastrointest Endosc 2011;74:176-181.

8. Moon JH, Choi HJ, Kim DC, et al. A newly designed fully covered metal stent for lumen apposition in EUS-guided drainage and access: a feasibility study (with videos). Gastrointest Endosc 2014;79:990-995.

9. Chan JHY, Teoh AYB. Current status of endoscopic gallbladder drainage. Clin Endosc 2018;51:150-155.

10. Jang JW, Lee SS, Song TJ, et al. Endoscopic ultrasound-guided transmural and percutaneous transhepatic gallbladder drainage are comparable for acute cholecystitis. Gastroenterology 2012;142:805-811.

11. Kwan V, Eisendrath P, Antaki F, Le Moine O, Devière J. EUS-guided cholecystenterostomy: a new technique (with videos). Gastrointest Endosc 2007;66:582-586.

12. Lee SS, Park DH, Hwang CY, et al. EUS-guided transmural cholecystostomy as rescue management for acute cholecystitis in elderly or high-risk patients: a prospective feasibility study. Gastrointest Endosc 2007;66:1008-1012.

13. Song TJ, Park DH, Eum JB, et al. EUS-guided cholecystoenterostomy with single-step placement of a 7F double-pigtail plastic stent in patients who are unsuitable for cholecystectomy: a pilot study (with video). Gastrointest Endosc 2010;71:634-640.

14. Kjaer DW, Kruse A, Funch-Jensen P. Endoscopic gallbladder drainage of patients with acute cholecystitis. Endoscopy 2007;39:304-308.

15. Mutignani M, Iacopini F, Perri V, et al. Endoscopic gallbladder drainage for acute cholecystitis: technical and clinical results. Endoscopy 2009;41:539-546.

16. Pannala R, Petersen BT, Gostout CJ, Topazian MD, Levy MJ, Baron TH. Endoscopic transpapillary gallbladder drainage: 10-year single center experience. Minerva Gastroenterol Dietol 2008;54:107-113. 